\section{Percepción del paciente ante el acto anestésico: desarrollo de una encuesta breve en español para medir satisfacción}

\author{
Patient perception of the anesthetic act: development of \\ a brief survey in Spanish to measure satisfaction
}

\author{
Dr. Donal J Rocher-Hernández,* Dra. Eva María Luna-Rivera ${ }^{\ddagger}$
}

Citar como: Rocher-Hernández DJ, Luna-Rivera EM. Percepción del paciente ante el acto anestésico: desarrollo de una encuesta breve en español para medir satisfacción. Rev Mex Anestesiol. 2021; 44 (4): 263-271. https://dx.doi. org/10.35366/100871

RESUMEN. Introducción: El desarrollo de encuestas representa un proceso complejo que requiere la verificación de su utilidad antes de su aplicación. A nivel internacional existen pocos instrumentos para medir la percepción de la atención anestésica en cirugías electivas, sin olvidar que, en su mayoría, se crearon en inglés; por lo que el propósito de este estudio fue desarrollar una encuesta breve sobre la atención anestésica como un indicador de calidad en la población mexicana. Material y métodos: Se trata de un estudio cualitativo que implica la fase de la construcción de una encuesta en la que se realizó una revisión de la literatura internacional y local de los instrumentos para medir satisfacción en anestesiología. Posteriormente, se realizaron entrevistas informales aleatorias a 82 pacientes sobre temas asociados al acto anestésico para la construcción de ítems. Resultados: La propuesta incluye 16 ítems divididos en tres secciones correspondientes a las tres valoraciones anestésicas básicas en una cirugía electiva y que exploran seis dominios: trato adecuado, comunicación, autonomía, atención oportuna, efectos secundarios y satisfacción. Conclusiones: La propuesta presentada es un potencial instrumento que podría reflejar la calidad percibida de la atención anestésica en nuestra población, por lo que, para fortalecer este proceso, se requerirá continuar con sus respectivas validaciones.

ABSTRACT. Introduction: Survey development represents a complex process that requires verification of its usefulness before its application. At an international level, there are few instruments to measure the perception of anaesthetic care in elective surgeries, without forgetting that most of them were created in English; therefore, the purpose of this study was to develop a brief survey on anaesthetic care as an indicator of quality in the Mexican population. Material and methods: This is a qualitative study that involves the construction phase of a survey in which a review of the international and local literature of the instruments to measure satisfaction in anesthesiology was carried out. Subsequently, informal random interviews were conducted with 82 patients on the most sensitive issues associated with the anaesthetic act for the construction of items. Results: The proposal consists of 16 items divided into three sections corresponding to the three basic anaesthetic evaluations in elective surgery and that explore five domains: communication, autonomy, timely care, adequate treatment, and satisfaction. Conclusions: The proposal made in this project is a potential instrument that could reflect the perceived quality of anaesthetic care in our population, so to strengthen this process it will be necessary to continue with their respective validations.

\section{INTRODUCCIÓN}

$\mathbf{P}$ ara evaluar la calidad de la atención médica se deben considerar los conceptos: calidad clínica y calidad percibida. La primera involucra la forma en que el proveedor de los servicios de salud ejerce sus insumos para resolver y mejorar la salud del paciente de manera efectiva, basada en evidencias y sin sub- ni sobreutilizar recursos ${ }^{(1)}$. Mientras que la calidad percibida se enfoca en la opinión del paciente y representa la evaluación de su experiencia basada en sus valores, cultura, rumores e interacciones con el entorno médico que suele reflejarse a través de la satisfacción ${ }^{(1-4)}$.

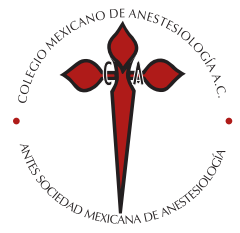

Palabras clave: Calidad de atención anestésica, calidad percibida, satisfacción en anestesia.

Keywords:

Quality of anesthetic care, quality perception, satisfaction in anesthetic care.

\section{* Médico residente de Anestesiología. \\ ‡ Departamento de Enseñanza e Investigación.}

Hospital Central Sur de Alta Especialidad de Petróleos Mexicanos. Ciudad de México, México.

\section{Correspondencia:}

Eva María Luna-Rivera MD, MSc \& PhD.

Anillo Periférico, Blvd. Adolfo Ruiz Cortines Núm. 4091, Col. Fuentes del Pedregal, 14140, Alcaldía Tlalpan, Ciudad de México. Tel: 55 5645-1684, ext. 51168

E-mail: eva_m_luna@ yahoo.com.mx https://orcid.org/00000002-1135-116X

Recibido: 20-10-2020

Aceptado: 06-04-2021

Abreviaturas:

EQUATOR = Enhancing the QUAlity and Transparency Of health Research. ISAS = Iowa Satisfaction with Anesthesia Scale. LPPSq = Leiden Perioperative Care Patient Satisfaction Questionnaire. 
Para el anestesiólogo la percepción del paciente acerca del acto anestésico representa a menudo un problema, derivado principalmente de la falta de información ${ }^{(5)}$. Es frecuente que los pacientes refieran angustia y miedo; situación que podría resolverse al ofrecer información clara e involucrar al paciente en la toma de decisiones; se ha demostrado que esto influye en la coordinación exitosa de la atención perioperatoria ${ }^{(6)}$. De no llevarse a cabo esta interacción médico-paciente, corremos el riesgo de que se desencadene una respuesta neuroendocrina que aumente el riesgo de morbimortalidad durante su estancia intrahospitalaria ${ }^{(7-9)}$.

Entre los métodos más empleados para conocer el nivel de satisfacción del usuario están los buzones de quejas o sugerencias, los cuestionarios y las encuestas. El desarrollo de estos instrumentos de medición, especialmente las encuestas, representa un proceso complejo y largo que requiere la comprobación de su utilidad previo a su implementación.
A nivel internacional existen pocos instrumentos con una construcción y validación estricta para medir la percepción de la calidad de la atención anestésica ${ }^{(10,11)}$, además de que sus indicadores son difíciles de homologar, sin olvidar que, en su mayoría, están diseñados en inglés; situación que exige la propuesta de nuevas herramientas en anestesiología que evalúen la percepción del paciente y con ello implementar estrategias para mejorar la calidad de la atención.

En México, durante décadas, se han desarrollado diversos programas nacionales para mejorar la calidad de la atención, enfocándose principalmente a la calidad clínica; ejemplo de ello son las certificaciones de hospitales y la expansión de los servicios de salud, programas que no necesariamente conllevan a la mejora en la calidad percibida. Más aún, a pesar de que desde hace más de dos décadas contamos con un organismo que evalúa y vigila la calidad de la atención médica (CONAMED, 1996), éste se enfoca más a las violaciones en

Tabla 1: Instrumentos de medición propuestos en México para medir la calidad de la atención médica.

\begin{tabular}{|c|c|c|c|}
\hline Organismo & Nombre & Objetivo & Áreas de evaluación \\
\hline $\begin{array}{l}\text { Instituto Nacional de Salud Públi- } \\
\text { ca-Secretaría de Salud, 2016(13) }\end{array}$ & $\begin{array}{l}\text { Encuesta nacional de salud y nutri- } \\
\text { ción de medio camino } 2016 \\
\text { Cuestionario de calidad de la } \\
\text { atención del paciente diabético, con } \\
\text { hipertensión o dislipidemia }\end{array}$ & $\begin{array}{l}\text { Medir la percepción de calidad de la } \\
\text { atención del paciente diabético, con } \\
\text { hipertensión o dislipidemia }\end{array}$ & $\begin{array}{l}\text { - Prestadores de servicios } \\
\text { - Accesibilidad y calidad } \\
\text { - Medicamentos } \\
\text { - Laboratorios y gabinete } \\
\text { - Satisfacción-calidad de la atención }\end{array}$ \\
\hline $\begin{array}{l}\text { Comisión Nacional de Protección } \\
\text { Social en Salud-Secretaría de } \\
\text { Salud, 2016(14) }\end{array}$ & $\begin{array}{l}\text { Estudio de satisfacción de usuarios } \\
\text { al SPSS }\end{array}$ & $\begin{array}{l}\text { Medir la percepción de calidad } \\
\text { de la atención médica y servicios } \\
\text { de salud que recibe la población } \\
\text { afiliada } \\
\text { Identificar los problemas de calidad } \\
\text { en los servicios que reciben }\end{array}$ & $\begin{array}{l}\text { - Grado de satisfacción y percep- } \\
\text { ción de la calidad de los servicios } \\
\text { - Satisfacción con la atención } \\
\text { médica recibida } \\
\text { - Calificación de la calidad } \\
\text { - Calificación del trato recibido } \\
\text { - Problemas de calidad percibidos }\end{array}$ \\
\hline $\begin{array}{l}\text { Dirección General de Calidad y } \\
\text { Educación en Salud-Secretaría de } \\
\text { Salud, 2015(15) }\end{array}$ & $\begin{array}{l}\text { Encuesta de satisfacción trato } \\
\text { adecuado y digno }\end{array}$ & $\begin{array}{l}\text { Conocer el desempeño de las uni- } \\
\text { dades de salud con relación al trato } \\
\text { adecuado y digno }\end{array}$ & $\begin{array}{l}\text { - Trato digno } \\
\text { - Atención oportuna } \\
\text { - Comunicación } \\
\text { - Autonomía } \\
\text { - Financiamiento } \\
\text { - Satisfacción }\end{array}$ \\
\hline $\begin{array}{l}\text { Instituto de Seguridad y Servicios } \\
\text { Sociales de los Trabajadores del } \\
\text { Estado. Centro de Investigación en } \\
\text { Evaluación y Encuestas del Instituto } \\
\text { Nacional de Salud Pública de Méxi- } \\
\text { co, } 2012^{(16)}\end{array}$ & $\begin{array}{l}\text { Encuesta de salud y nutrición del } \\
\text { derechohabiente del ISSSTE }\end{array}$ & $\begin{array}{l}\text { Contar con información confiable, } \\
\text { precisa y generalizable sobre los } \\
\text { aspectos de salud y nutrición de } \\
\text { mayor relevancia por su prevalencia } \\
\text { observada, tanto en enfermedades } \\
\text { crónicas como agudas transmisi- } \\
\text { bles, entre la población derechoha- } \\
\text { biente de todo el país }\end{array}$ & $\begin{array}{l}\text { - Características demográficas } \\
\text { - Prevalencia de enfermedades } \\
\text { transmisibles y no transmisibles } \\
\text { - Características de la atención } \\
\text { - Grado de satisfacción } \\
\text { - Estado de nutrición de la población }\end{array}$ \\
\hline $\begin{array}{l}\text { Instituto Mexicano del Seguro } \\
\text { Social, 2009(17) }\end{array}$ & $\begin{array}{l}\text { Encuesta nacional de satisfacción } \\
\text { a derechohabientes usuarios de } \\
\text { servicios médicos }\end{array}$ & $\begin{array}{l}\text { Conocer el nivel de satisfacción de } \\
\text { los usuarios con los servicios médi- } \\
\text { cos de los tres niveles de atención } \\
\text { que presta el IMSS }\end{array}$ & $\begin{array}{l}\text { - Satisfacción } \\
\text { - Trato } \\
\text { - Surtimiento de medicamentos } \\
\text { - Servicio de urgencias } \\
\text { - Limpieza }\end{array}$ \\
\hline $\begin{array}{l}\text { Fundación Mexicana para la Salud, } \\
1994 \text { y } 2000^{(18)}\end{array}$ & $\begin{array}{l}\text { Encuesta nacional de satisfacción } \\
\text { con los servicios de salud }\end{array}$ & $\begin{array}{l}\text { Conocer la satisfacción de la po- } \\
\text { blación mexicana con los servicios } \\
\text { de salud }\end{array}$ & Datos no disponibles \\
\hline
\end{tabular}

SPSS = Sistema de Protección Social en Salud; ISSSTE = Instituto de Seguridad y Servicios Sociales para los Trabajadores del Estado.

Creada por: Donal J Rocher-Hernández y Eva M Luna-Rivera. 
las normas de calidad a partir de las quejas emitidas por la insatisfacción de los pacientes (Tabla 1) ${ }^{(12)}$.

Por último, ninguno de los programas nacionales considera de manera específica la atención anestésica, por lo que el objetivo del presente trabajo está enfocado en la primera fase cualitativa de la construcción de un instrumento corto capaz de evaluar la «atención anestésica percibida» mediante una encuesta de satisfacción en idioma español en un lenguaje claro y simple. Este objetivo incluye la revisión bibliográfica exhaustiva, entrevistas aleatorias a pacientes y la construcción de ítems que posteriormente requerirán de las fases de validación.

\section{MATERIAL Y MÉTODOS}

\section{Diseño del estudio}

Es un estudio cualitativo, dirigido a la construcción de un instrumento para evaluar la calidad percibida de la atención anestésica, que incluye una revisión exhaustiva de la literatura y entrevistas aleatorias a pacientes para la construcción de ítems. Este manuscrito se adhiere a las pautas aplicables para mejorar la calidad y transparencia de la investigación en salud (EQUATOR).

\section{Requisitos éticos}

Este proyecto fue aprobado por los Comités de Investigación y Ética de Investigación del Hospital Central Sur de Alta Especialidad de Petróleos Mexicanos con registro 02/2019. El consentimiento informado de las entrevistas informales se obtuvo libremente.

\section{Fase cualitativa y cuantitativa para el desarrollo de una encuesta}

Para desarrollar una encuesta debemos seguir una serie de pasos que comienzan con una revisión exhaustiva de la literatura que nos dará la certeza de que no existe un instrumento como el propuesto, o que existe, pero el contexto está parcialmente definido, lo que justifica la elaboración de una nueva propuesta ${ }^{(9)}$. Debemos definir claramente el concepto que queremos evaluar, ya que el objeto de medición puede definirse en diferentes perspectivas teóricas y, por ende, tener diferentes definiciones. Esto es, un instrumento de medición apropiado debe ser capaz de registrar datos que representen los conceptos o variables que tenemos en mente.

Una vez creados los elementos, estarán sujetos a validación en dos fases: la primera es cualitativa e implica la validez de contenido, y la segunda es cuantitativa y se refiere a la confiabilidad, validez de constructo y de criterio (Figura 1)(19-23). Para la fase cualitativa, o validez de contenido, existen varias metodologías, las más utilizadas son las entrevistas cognitivas o la validación por parte de jueces. Las entrevistas cognitivas nos brindan datos cualitativos en los que podemos profundizar y obtener una imagen completa del problema del estudio. Mientras que, en la validación de los jueces, un grupo de expertos en el área o en diferentes campos del conocimiento determina la coherencia de los ítems y la complejidad cognitiva ${ }^{(19-22)}$. Si los jueces dan su aprobación a favor, los ítems dejan de llamarse así y desde entonces se considera como un instrumento.

Hasta ahora, terminamos la fase cualitativa de construcción, y podemos decir que nuestro instrumento tiene validez de contenido. A partir de este punto, comienza la fase cuantitativa del proceso de validación y su respectivo análisis estadístico $^{(23,24)}$.

\section{Revisión de la literatura y validez racional}

Para una validez racional realizamos una revisión de la literatura nacional e internacional sobre la calidad percibida de la atención anestésica para conocer su estado del arte. Para este propósito se emplearon los términos MeSH: Health care survey/Health care quality/Quality indicators AND Anesthesia; en español: percepción de la atención, satisfacción del paciente, calidad de la atención y validación de escalas, asociados con las palabras anestesia/anestesiología. El período de búsqueda duró del 5 al 20 de enero de 2019. Seleccionamos principalmente aquellos estudios publicados en los últimos 10 años, o más, en el caso de estudios muy relevantes.

\section{Validez de contenido y propuesta de dominios y elementos de selección}

Para la validez del contenido iniciamos con entrevistas informales aleatorias durante un mes a pacientes programados para cirugía electiva y sin antecedentes de anestesia previa que asistieron a su primera evaluación preanestésica en consulta externa, días antes de su cirugía. Les realizamos tres preguntas básicas: ¿Sabes cuál es la anestesia que le necesitamos realizar? ¿Cuáles son sus principales dudas, inquietudes y expectativas sobre la anestesia y la atención del anestesiólogo? ¿Qué podríamos hacer para que se sienta seguro(a) y tranquilo(a) durante el procedimiento? A partir de las respuestas emitidas preparamos una lista de los conceptos principales para hacer una comparación con los elementos elegidos durante la revisión de la literatura y elegir aquéllos que fueron más representativos para la construcción preliminar del instrumento.

\section{RESULTADOS}

\section{Revisión de la literatura y validez racional}

Obtuvimos 69 artículos por revisión de la literatura; 38 de ellos fueron excluidos durante la revisión, ya que no cumplieron 

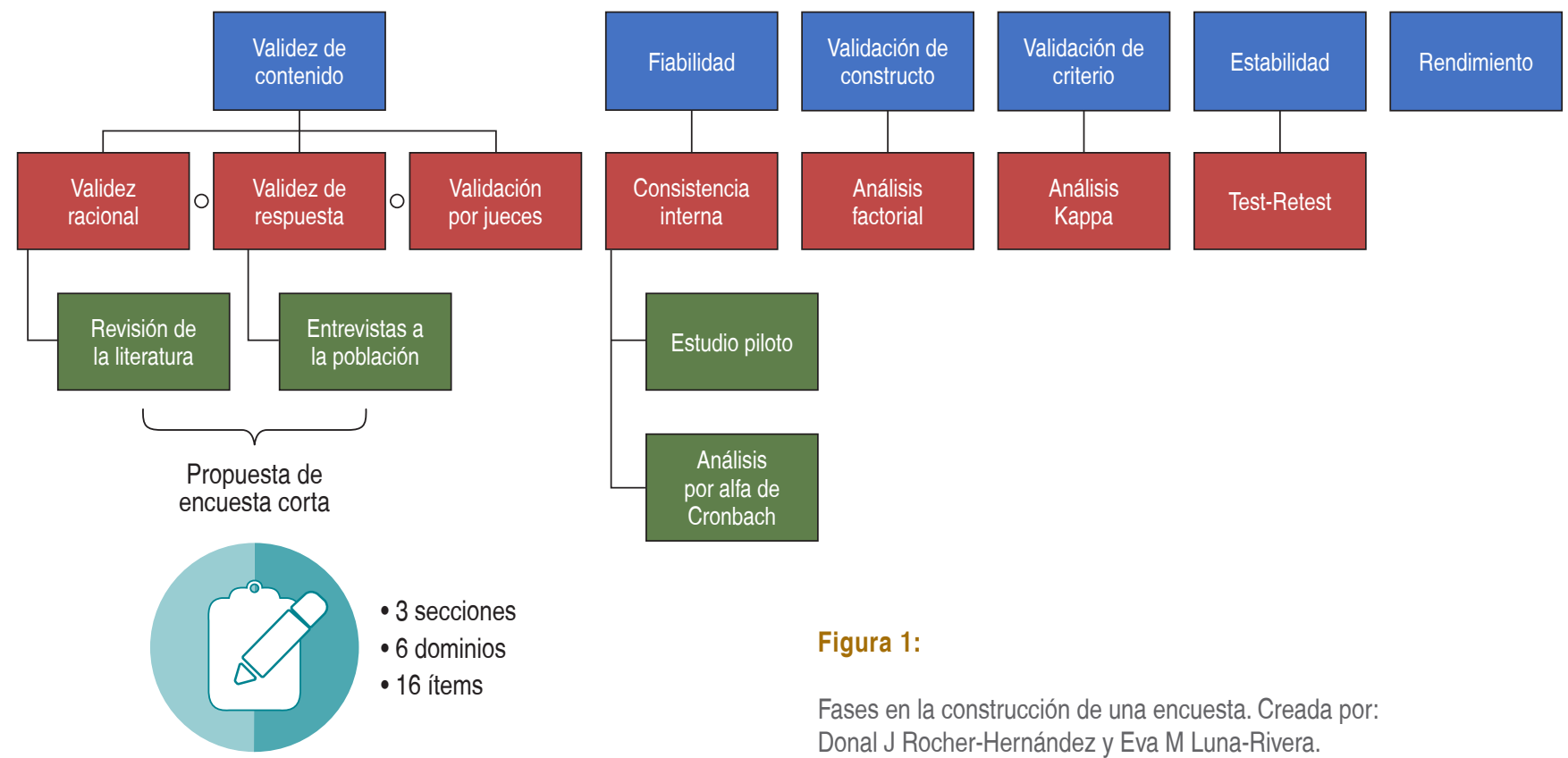

Figura 1:

Fases en la construcción de una encuesta. Creada por: Donal J Rocher-Hernández y Eva M Luna-Rivera.

\begin{tabular}{|c|c|c|c|}
\hline Preguntas & Respuestas agrupadas ( $\mathrm{N}=82$ ) & $\%$ & Dominio que podría resolver el problema \\
\hline \multirow{6}{*}{$\begin{array}{l}\text { ¿Sabe en qué consiste la anestesia? } \\
\text { ¿Cuáles son sus mayores dudas o temores } \\
\text { de la anestesia? }\end{array}$} & Desconocen el procedimiento anestésico & 85 & \multirow[t]{3}{*}{ Mejorar la comunicación } \\
\hline & Temen: & & \\
\hline & No despertar & 77 & \\
\hline & Presentar una secuela & 15 & \multirow{3}{*}{$\begin{array}{l}\text { Mejorar la comunicación y la atención } \\
\text { oportuna }\end{array}$} \\
\hline & Tener efectos secundarios & 20 & \\
\hline & Sentir dolor durante y en el postoperatorio & 82 & \\
\hline \multirow{4}{*}{$\begin{array}{l}\text { ¿Qué podríamos hacer como médicos para } \\
\text { que usted sienta confianza y tranquilidad } \\
\text { durante el procedimiento? }\end{array}$} & Explicar claramente el procedimiento & 76 & \multirow{4}{*}{ Mejorar la comunicación y la autonomía } \\
\hline & Tratar con paciencia y respeto & 64 & \\
\hline & Plática reconfortante & 54 & \\
\hline & No saben & 21 & \\
\hline
\end{tabular}

Creada por: Donal J Rocher-Hernández y Eva M Luna-Rivera.

con el objetivo de este estudio, por lo tanto, nuestra selección final incluyó 31 artículos.

\section{Validez de respuesta y selección de dominios a explorar}

Durante un mes, logramos realizar 82 entrevistas informales aleatorias a pacientes para explorar los temas más sensibles alrededor de la anestesia. Obtuvimos múltiples respuestas que agrupamos en temas principales y que en su mayoría expresaban diversos «temores ante el acto anestésico» (Tabla 2).

Esta información en conjunto con la obtenida de la búsqueda bibliográfica nos permitió crear, modificar y agrupar los ítems en los seis dominios propuestos para este instrumento, que consideramos exploran de manera adecuada la percepción del paciente.

Los dominios son: 1. Trato adecuado; valora la actitud del personal hacia el paciente y sus familiares. 2. Comu- 
Tabla 3: Características de los ítems propuestos.

No.

ítem
Definición conceptual
Opciones de respuesta

Dominio explorado
Sección I. Primera consulta con el anestesiólogo (valoración preanestésica en el consultorio)

$1 \quad$ Durante su primera consulta antes de la cirugía, ¿cómo le pareció el tiempo que tuvo que esperar desde la hora de su cita hasta ser atendido por un anestesiólogo?

2 ¿Considera usted que los médicos del servicio de anestesiología lo atendieron con respeto?

3 ¿El anestesiólogo le explicó en qué consistiría la anestesia, así como sus riesgos y beneficios?

4 ¿Qué tan clara le pareció la explicación?

$5 \quad$ ¿El anestesiólogo le dio la oportunidad de hacer preguntas o platicar de sus preocupaciones acerca de la anestesia?

$\begin{array}{ll}\text { Muy corto } & \text { Atención oportuna } \\ \text { Corto } & \\ \text { No recuerdo } & \\ \text { Largo } & \\ \text { Excesivo } & \text { Trato adecuado } \\ \text { Totalmente de acuerdo } & \\ \text { De acuerdo } & \\ \text { Ni de acuerdo ni en } & \\ \text { desacuerdo } & \\ \text { En desacuerdo } & \\ \text { Totalmente en desacuerdo } & \\ \text { Sí } & \\ \text { No recuerdo } & \\ \text { No } & \\ \text { Muy clara } & \\ \text { Clara } & \\ \text { No recuerdo } & \\ \text { Poco clara } & \\ \text { Nada clara } & \\ \text { Sí } & \\ \text { No recuerdo } & \\ \text { No } & \end{array}$

Sección II. Valoración del anestesiólogo justo antes de la cirugía en la sala de preoperatorio

6 ¿Cómo le pareció el tiempo que tuvo que esperar desde que llegó al área de preparación para su cirugía hasta ser valorado por un anestesiólogo?

$7 \quad$ ¿Considera usted que los médicos del servicio de anestesiología lo atendieron con respeto?

$8 \quad$ ¿El anestesiólogo le explicó en qué consistiría la anestesia, así como sus riesgos y beneficios?

$9 \quad$ ¿Qué tan clara le pareció la explicación?

10 ¿El anestesiólogo le dio la oportunidad de hacer preguntas o platicar de sus preocupaciones acerca de la anestesia?

Muy corto

Atención oportuna

Corto

No recuerdo

Largo

Excesivo

Totalmente de acuerdo Trato adecuado

De acuerdo

Ni de acuerdo ni en

desacuerdo

En desacuerdo

Totalmente en desacuerdo

Sí

No recuerdo

No

Muy clara

Clara

No recuerdo

Poco clara

Nada clara

Sí

No recuerdo

No

11 Marque con una $\mathrm{X}$ el malestar que haya tenido durante su estancia en el área de recuperación:

En caso de que haya presentado algún malestar, ¿el anestesiólogo se acercó a usted para preguntarle de su molestia?

\author{
Náusea y/o vómito \\ Dolor \\ Nerviosismo \\ Mareo \\ Ningún síntoma \\ Otros: \\ Sí Comunicación \\ No recuerdo \\ No
}

Comunicación

Comunicación

Autonomía

Indicador que evalúa el tiempo de espera por parte del usuario

Indicador que evalúa la percepción del usuario con respecto al trato que recibió durante la atención

Indicador que evalúa la calidad de la información otorgada por el personal de anestesiología

Indicador que evalúa la calidad de la información otorgada por el personal de anestesiología

Indicador que evalúa el trato al paciente durante la toma de decisiones

Indicador que evalúa el tiempo de espera por parte del usuario

Indicador que evalúa la percepción del usuario con respecto al trato que recibió durante la atención

Indicador que evalúa la calidad de la información otorgada por el personal de anestesiología

Indicador que evalúa la calidad de la información otorgada por el personal de anestesiología

Indicador que evalúa el trato al paciente durante la toma de decisiones

Indicador
Indicador que evalúa la calidad de la información otorgada por el personal de anestesiología 
Continúa la Tabla 3: Características de los ítems propuestos.

\begin{tabular}{|c|c|c|c|c|}
\hline $\begin{array}{l}\text { No. } \\
\text { ítem }\end{array}$ & Definición conceptual & Opciones de respuesta & Dominio explorado & Definición del dominio \\
\hline 13 & $\begin{array}{l}\text { ¿Cómo le pareció el tiempo que tuvo } \\
\text { que esperar para que resolvieran sus } \\
\text { molestias? }\end{array}$ & $\begin{array}{l}\text { Muy corto } \\
\text { Corto } \\
\text { No recuerdo } \\
\text { Largo } \\
\text { Excesivo }\end{array}$ & Atención oportuna & $\begin{array}{l}\text { Indicador que evalúa el tiempo de espe- } \\
\text { ra por parte del usuario }\end{array}$ \\
\hline 14 & $\begin{array}{l}\text { ¿Cómo le pareció el tiempo que } \\
\text { permaneció en el área de recuperación } \\
\text { hasta su traslado a una habitación o su } \\
\text { alta (en caso de que haya sido una cirugía } \\
\text { ambulatoria)? }\end{array}$ & $\begin{array}{l}\text { Muy corto } \\
\text { Corto } \\
\text { No recuerdo } \\
\text { Largo } \\
\text { Excesivo }\end{array}$ & Atención oportuna & $\begin{array}{l}\text { Indicador que evalúa el tiempo de espe- } \\
\text { ra por parte del usuario }\end{array}$ \\
\hline 15 & $\begin{array}{l}\text { ¿Qué tan satisfecho o satisfecha está con } \\
\text { la atención médica recibida por parte del } \\
\text { servicio de anestesiología? }\end{array}$ & $\begin{array}{l}\text { Muy satisfecho(a) } \\
\text { Satisfecho(a) } \\
\text { Poco satisfecho(a) } \\
\text { Nada satisfecho(a) }\end{array}$ & Satisfacción & $\begin{array}{l}\text { Indicador que evalúa la calidad del } \\
\text { servicio de anestesiología }\end{array}$ \\
\hline 16 & $\begin{array}{l}\text { ¿Qué podríamos hacer para mejorar la } \\
\text { calidad de la atención en el servicio de } \\
\text { anestesiología? }\end{array}$ & Libre & Satisfacción & $\begin{array}{l}\text { Indicador que evalúa la calidad del } \\
\text { servicio de anestesiología }\end{array}$ \\
\hline
\end{tabular}

Creada por: Donal J Rocher-Hernández y Eva M Luna-Rivera.

nicación; evalúa la calidad de la información dada al paciente y el tiempo brindado para aclarar sus dudas sobre su procedimiento. 3. Autonomía; valora la oportunidad que se le da al paciente para intervenir en la toma de decisiones relacionadas con su salud. 4. Atención oportuna; se refiere al tiempo transcurrido entre buscar atención y recibirla. 5 . Efectos secundarios: evalúa la presencia de efectos secundarios anestésicos y 6. Satisfacción; evalúa la percepción de los servicios recibidos con respecto a las expectativas del usuario.

\section{Propuesta de ítems}

Con los dominios ya definidos nos adherimos a las recomendaciones reportadas para la construcción adecuada de ítems ${ }^{(3)}$; entendiendo como un ítem a la unidad básica de información de un instrumento de evaluación que, por lo general, está constituido por preguntas cerradas. Construimos 16 ítems distribuidos en tres secciones correspondientes a las tres evaluaciones anestésicas necesarias en cirugía electiva: la primera evaluación o consulta preanestésica, realizada días antes de la cirugía; la segunda evaluación en la sala preoperatoria, justo antes de la cirugía; y la tercera evaluación en el área de recuperación, al final de la cirugía. Repetimos los ítems 2, 3, 4 y 5 en la sección II, y los ítems 1 y 6 son equivalentes a medida que explora la atención oportuna de los momentos prequirúrgicos (Tabla 3). Las opciones de respuesta se generaron en función de la escala Likert para obtener una evaluación cuantitativa en el futuro proceso de validación.

\section{DISCUSIÓN}

En el presente trabajo proponemos una breve encuesta en español para explorar la percepción del paciente sobre la atención anestésica, que puede ser muy útil para los servicios de anestesiología que buscan medir sus procesos de mejora continua en la calidad de la atención. A diferencia de otros instrumentos de anestesiología que exploran indicadores de calidad clínica que dependen del proveedor o el servicio de salud $^{(3,25)}$, proponemos elementos que consideramos factores completamente modificables por los anestesiólogos, considerando que la relación médico-paciente en los países en desarrollo por lo general tiene mayor peso para evaluar la satisfacción ${ }^{(4)}$.

Se trata de una herramienta compuesta de tres secciones que corresponden a los tres momentos en que se recibe la atención anestésica para cirugías electivas: la primera consulta, que puede ser días previos a la cirugía; la segunda consulta, previo a la cirugía con el paciente ya hospitalizado; y la última en el área de recuperación, posterior a la cirugía. A pesar de que la encuesta se divide en estos tres momentos, no significa que debamos solicitar que el paciente responda en cada momento anestésico. Sugerimos que el paciente responda la encuesta cuando se encuentre libre de efectos anestésicos y lo más confortable posible, disminuyendo de esta forma la existencia de factores que pudieran alterar su percepción ante la atención que ha recibido. En ese momento nosotros le solicitaremos que recuerde los tres momentos en los que fue valorado por el anestesiólogo y con base en ello responda la encuesta. 
Nuestra propuesta incluye seis dominios que consideramos son los más relevantes y en los que puede incidir el anestesiólogo. Sin duda, la comunicación médico-paciente tiene un peso sustancial que logramos observar durante las entrevistas informales, por lo que incluimos preguntas que exploran este dominio en las tres secciones. Además, debido a que muchos pacientes suelen recibir por diversas causas sólo dos valoraciones (justo antes de la cirugía y después de ella), se podría emplear la herramienta desde la sección II, cuyas preguntas son iguales o equivalentes. Los ítems se redactaron a manera de preguntas con opciones de respuesta mediante escala tipo Likert para su posterior evaluación cuantitativa $^{(26)}$.

Algunos países cuentan con instrumentos estándar para medir satisfacción como: Picker Patient Experience Questionnaire-15 (PPE-15) en Reino Unido, el Hospital Consumer Assessment of Healthcare Providers and Systems (HCAHPS) en Estados Unidos y Victorian Patient Satisfaction Monitor (VPSM) empleado en Australia ${ }^{(3)}$. Todos ellos con dominios esenciales enfocados en la atención y percepción del paciente sin ser específicos en la atención anestésica.

En México también hemos desarrollado esfuerzos dirigidos a mejorar la atención primaria centrada en el paciente como modelo de calidad de la atención médica a través de la percepción de los usuarios ${ }^{(27-37)}$. Hace poco más de dos décadas se reportaron los resultados de la Encuesta Nacional de Salud aplicada en 1994 con el objetivo de investigar la percepción de la calidad de la atención de los servicios de salud. Esta encuesta se aplicó a más de 3,000 mexicanos que acudieron a diversos servicios de salud públicos y privados de forma ambulatoria, explorando, en su mayoría, dominios dependientes del proveedor de servicios de salud como accesibilidad, costos, organización, estructura, materiales, equipos, procesos, entre otros. El único dominio explorado no dependiente del proveedor fue el trato por el personal de salud. El resultado final concluyó que, en general, más del $80 \%$ consideró haber recibido buena atención y como motivos principales para no regresar al servicio fueron los tiempos largos de espera y la mala atención al usuario ${ }^{(27)}$.

Un reporte adicional en 2004 relaciona directamente la satisfacción con las expectativas del usuario, menciona que el trato otorgado por el médico constituye un elemento fundamental en la satisfacción de sus expectativas, lo cual refleja la importancia de la relación médico-paciente, aun cuando otros factores dependientes de la institución sean deficientes $^{(28)}$.

Diversos reportes en México han explorado la satisfacción del usuario a través de instrumentos nacionales ${ }^{(27-30,32-34)} \mathrm{o}$ internacionales ${ }^{(31)}$ validados para nuestra población que, en su gran mayoría, exploran aspectos dependientes de la institución prestadora de servicios. Aquellos instrumentos que evalúan la relación médico-paciente, tales como habilidades interpersonales, comunicación, tratamiento digno, autonomía, elección y actitud de los profesionales de la salud, consideran que estos aspectos son factores fundamentales para obtener resultados de satisfacción positivos o negativos ${ }^{(28,31-35)}$.

Al realizar un análisis de los instrumentos internacionales más recomendados para evaluar la atención anestésica como ISAS, Quality of Preanesthetic Visit, Perioperative Questionnaire, English Adaptation of the LPPSq y Heidelberg Perianesthetic Questionnaire, observamos que tienen algunas limitaciones, ya que su aplicación se sugiere en momentos anestésicos específicos, o bien para fines de investigación ${ }^{(20)}$. Por ejemplo, la escala ISAS (Iowa Satisfaction with Anesthesia Scale), que contiene 11 ítems con cinco posibilidades de respuesta basadas en la escala Likert, ha sido validada al español; sin embargo, enfatiza mucho en los efectos secundarios de la anestesia sin abarcar más dominios de la relación médico-paciente, por lo que consideramos que su interpretación es limitada ${ }^{(25)}$.

En México hemos explorado poco en la satisfacción del usuario en el cuidado anestésico, encontrando sólo un par de reportes $^{(38-40)}$, como el de Ugalde y colaboradores en 2012 que evaluaron la satisfacción preanestésica a través de un instrumento de 12 reactivos que exploraba cuatro dominios, dos asociados con aspectos dependientes del prestador de servicios médicos y dos referentes a la actitud y claridad de comunicación del médico, los cuales fueron evaluados con un porcentaje alto de satisfacción. Sin embargo, no mencionan con claridad la estructura de las preguntas ni las opciones de respuesta a cada ítem y sus resultados se concretan a ser porcentuales $^{(39)}$.

Otro ejemplo es el instrumento propuesto en México por Torres-González y colegas, que contiene ocho preguntas y una evaluación global, que a pesar de tocar dominios importantes no explora la autonomía ni la atención oportuna al paciente, además de que sus posibles respuestas se reducen a contestar «Sí» o «No» ${ }^{(40)}$. Para este tipo de instrumentos que miden actitudes, se recomienda seguir escalas tipo Likert $^{(10,19,20,26,36)}$.

Hasta este punto del desarrollo del instrumento, consideramos que nuestra propuesta presenta múltiples ventajas como: ser corto, autoaplicable, considera múltiples dominios y que podría aplicarse en diversos momentos anestésicos. Sin embargo, también presenta algunas limitaciones, ya que no explora la calidad clínico-técnica, que suele depender de la institución de salud y tampoco explora la atención de otros profesionales de la salud que podrían estar involucrados en la atención anestésica.

Nuestro siguiente objetivo es continuar con las validaciones complementarias como la validación por jueces y la 
cuantitativa con el fin de valorar su fiabilidad o consistencia interna y su validez en otros niveles de investigación como la de criterio y de constructo.

\section{CONCLUSIONES}

Aunque existen diversos instrumentos propuestos a nivel internacional para medir la satisfacción anestésica, muy pocos cuentan con una construcción y validación estrictas y ninguno ha logrado abarcar de manera adecuada los aspectos que influyen en la percepción del paciente, por lo que se requiere de nuevas propuestas que exploren los aspectos más importantes de la percepción del paciente y que sigan una metodología precisa.

Financiamiento: La presente investigación no ha recibido ayudas específicas provenientes de agencias del sector público, sector comercial o entidades sin ánimo de lucro.

Conflicto de intereses: Ninguno. Los autores Donal J RocherHernández y Eva María Luna-Rivera declaran no tener ningún conflicto de intereses.

\section{REFERENCIAS}

1. Hanefeld J, Powell-Jackson T, Balabanova D. Understanding and measuring quality of care: dealing with complexity. Bull World Health Organ. 2017;95:368-374. Available from: http://dx.doi.org/10.2471/ BLT.16.179309

2. Oh J, Cho H, Kim YY, Park HJ, Kim HK. An integrative review on development of “QUality Of care Through the patients' Eyes” (QUOTE) instruments. J Nurs Care Qual. 2015;30:E26-E231. Available from: http://dx.doi.org/10.1097/NCQ.0000000000000127

3. Hawkins RJ, Swanson B, Kremer MJ, Fogg L. Content validity testing of questions for a patient satisfaction with general anesthesia care instrument. J Perianesth Nurs. 2014;29:28-35. Available from: http:// dx.doi.org/10.1016/j.jopan.2013.05.011

4. Alfred M, Ubogaya K, Chen X, Wint D, Worral PS. Effectiveness of culturally focused interventions in increasing the satisfaction of hospitalized Asian patients: a systematic review. JBI Database System Rev Implement Rep. 2016;14:219-256. Available from: http://dx.doi. org/10.11124/JBISRIR-2016-003048

5. García dYPMJ, Rodríguez SF, Carmona OL. Validación de cuestionarios. Reumatología Clínica. 2009;5:171-177. Disponible en: https://doi. org/10.1016/j.reuma.2008.09.007

6. Ortiz J, Wang S, Elayda MA, Tolpin DA. Preoperative patient education: can we improve satisfaction and reduce anxiety? Rev Bras Anestesiol. 2015;65:7-13. Available from: http://dx.doi.org/10.1016/j. bjan.2013.07.009

7. Vetter TR, Ivankova NV, Goeddel LA, McGwin G Jr, Pittet JF; UAB Perioperative Surgical Home Group. An analysis of methodologies that can be used to validate if a perioperative surgical home improves the patient-centeredness, evidence-based practice, quality, safety, and value of patient care. Anesthesiology. 2013;119:1261-1274. Available from: http://dx.doi.org/10.1097/ALN.0b013e3182a8e9e6

8. Jlala HA, French JL, Foxall GL, Hardman JG, Bedforth NM. Effect of preoperative multimedia information on perioperative anxiety in patients undergoing procedures under regional anaesthesia. $\mathrm{Br} \mathrm{J}$ Anaesth. 2010;104:369-374. Available from: https://doi.org/10.1093/bja/aeq002

9. Blitz JD, Kendale SM, Jain SK, Cuff GE, Kim JT, Rosenberg AD. Preoperative evaluation clinic visit associated with decreased risk of in-hospital postoperative mortality. Anesthesiology. 2016;125:280-294. Available from: http://doi.org/10.1097/ALN.0000000000001193

10. Chanthong P, Abrishami A, Wong J, Herrera F, Chung F. Systematic review of questionnaires measuring patient satisfaction in ambulatory anesthesia. Anesthesiology. 2009;110:1061-1067. Available from: http:// doi.org/10.1097/ALN.0b013e31819db079

11. Hocking G, Weightman WM, Smith C, Gibbs NM, Sherrard K. Measuring the quality of anaesthesia from a patient's perspective: development, validation, and implementation of a short questionnaire. Br J Anaesth. 2013;111:979-989. Available from: http://doi.org/10.1093/ bja/aet284
12. Aguirre GHG, Zavala VJA, Hernández-Torres F, Fajardo DG. Quality of medical care and patient surgical safety: medical error, malpractice and professional liability. Spanish. Cir Cir. 2010;78:456-462. Available from: https://www.medigraphic.com/pdfs/circir/cc-2010/cc105o.pdf

13. Secretaría de Salud. Encuesta Nacional de Nutrición de Medio Camino 2016. Cuestionario de calidad de la atención del paciente diabético, con hipertensión o dislipidemias. México: Instituto Nacional de Salud Pública; 2016. Disponible en: https://ensanut.insp.mx/ensanut2016/ Formatos\%20de\%20los\%20cuestionarios\%20con\%20variables/ F3_Enfermedades\%20cr\%C3\%B3nicas_Calidad\%20de\%20la\%20 atencion.pdf

14. Secretaría de Salud. Estudio de satisfacción de usuarios al SPSS. México: Comisión Nacional de Protección Social en Salud, Instituto Nacional de Salud Pública; 2016. Disponible en: http://www.ssm.gob.mx/portal/ pdf/Informe\%20Ejecutivo\%20SPSS\%202016.pdf

15. Secretaría de Salud. Encuesta de satisfacción trato adecuado y digno. México: Dirección General de Calidad y Educación en Salud; 2020. Disponible en: http://www.calidad.salud.gob.mx/site/calidad/encuesta_ satisfaccion_trato_digno.html

16. Instituto de Seguridad y Servicios Sociales de los Trabajadores del Estado. Encuesta de Salud y Nutrición de los Derechohabientes del ISSSTE 2011/2012. México: Centro de Investigación en Evaluación y Encuestas del Instituto Nacional de Salud Pública de México; 2012. Disponible en: http://www.issste.gob.mx/images/downloads/isssteduca/ cacym/6aSesionOrdinariaCACyM_ensader2012.pdf

17. Instituto Mexicano del Seguro Social. Encuesta nacional de satisfacción a derechohabientes usuarios de servicios médicos. Sistema integral de medición de la satisfacción de usuarios del IMSS. Disponible en: http://www.imss.gob.mx/sites/all/statics/pdf/estadisticas/ENSAT/2018/ NOV/2018_Nov_Ensat_Cuestionario_3erNivel.pdf

18. Knaul FM, Nigenda G. Caleidoscopio de Salud: De la investigación a las políticas y de las políticas a la acción. Capítulo 11: Opinión pública sobre el sistema salud en el 2000. México: Fundación Mexicana para la Salud; 2003. pp. 163-169. Disponible en: http://funsalud.org.mx/portal/ linea-editorial/libros-manuales-y-boletines/economia-y-salud/articulosen-revistas/

19. Beattie M, Murphy DJ, Atherton I, Lauder W. Instruments to measure patient experience of healthcare quality in hospitals: a systematic review. Syst Rev. 2015;4:97. Available from: http://doi.org/10.1186/s13643-015-0089-0

20. Barnett SF, Alagar RK, Grocott MP, Giannaris S, Dick JR, Moonesinghe SR. Patient-satisfaction measures in anesthesia: qualitative systematic review. Anesthesiology. 2013;119:452-478. Available from: http://doi. org/10.1097/ALN.0b013e3182976014

21. Suhonen R, Leino-Kilpi H, Valimaki M, Kim HS. The Patient Satisfaction Scale--an empirical investigation into the Finnish adaptation. J Eval Clin Pract. 2007;13:31-38. Available from: http://doi.org/10.1111/j.13652753.2006.00643.x 
22. Urrutia EM, Barrios AS, Gutiérrez NM, Mayorga CM. Métodos óptimos para determinar validez de contenido. Revista Cubana de Educación Médica Superior. 2014;28:547-558. Disponible en: https://www. medigraphic.com/pdfs/educacion/cem-2014/cem143n.pdf

23. Silberman MS, Moreno AL, Hernández MD, Martínez GA, Díaz LHO. Construcción y validación de un instrumento para medir la satisfacción de los pacientes del primer nivel de atención médica en la Ciudad de México. Gac Med Mex. 2016;152:43-50. Disponible en: https://www. medigraphic.com/pdfs/gaceta/gm-2016/gm161g.pdf

24. Cruz AA, Cruz PE. Metodología para la construcción de instrumentos de medición en salud. Alerg Asma e Inmunol Pediatr. 2017;26:100-105. Disponible en: https://www.medigraphic.com/pdfs/alergia/al-2017/ al173d.pdf

25. Jiménez GLF, del Real CA. Validación al español de la escala «The Iowa satisfaction with anesthesia scale (ISAS)» para cuidado anestésico monitorizado en cirugía de oftalmología. Rev Colomb Anestesiol. 2014;42:272-280. Disponible en: https://doi.org/10.1016/j. rca.2014.07.008

26. Phillips NM, Street M, Haesler E. A systematic review of reliable and valid tools for the measurement of patient participation in healthcare. BMJ Qual Saf. 2016;25:110-117. Available from: https://doi. org/10.1136/bmjqs-2015-004357

27. Ramírez STdJ; Nájera AP, Nigenda LG. Percepción de la calidad de la atención de los servicios de salud en México: perspectiva de los usuarios. Salud Pública de México. 1998;40:3-12. Disponible en: http:// saludpublica.mx/index.php/spm/article/view/6051/7047

28. Ortiz ERM, Muñoz JS, Torres CE. Satisfacción de los usuarios de 15 hospitales de Hidalgo, México. Rev Esp Salud Publica. 2004;78:527537. Dis ponible en: http://scielo.isciii.es/pdf/resp/v78n4/original5.pdf

29. Puentes RE, Gómez DO, Garrido LF. Trato a los usuarios en los servicios públicos de salud en México. Rev Panam Salud Publica. 2006;19:394402. Disponible en: https://doi.org/10.1590/s1020-49892006000600005

30. Sauceda VAL, Wirtz VJ, Santa-Ana TY, de la Luz Kageyama EM. Ambulatory health service users' experience of waiting time and expenditure and factors associated with the perception of low quality of care in Mexico. BMC Health Serv Res. 2010;10:178. Available from: https://doi.org/10.1186/1472-6963-10-178

31. Balderas PLM, Sat MD, Contreras HI, Solano MP, Hernández ChGA, Mariscal RI, et al. Análisis de la satisfacción con los cuidados en salud a través del cuestionario EORTC IN-PATSAT32 en pacientes con cáncer de mama, linfoma no hodgkin y cáncer colo-rectal en diferentes etapas clínicas. Relación con las características socio-demográficas, estados co- mórbidos y variables del proceso de atención en el Instituto Mexicano del Seguro Social. Value Health. 2011;14:S96-S99. Disponible en: https:// doi.org/10.1016/j.jval.2011.05.026

32. García OMS, Gómez AE, Díaz QG. Satisfacción del usuario como un indicador de calidad en el Servicio de Cirugía Cardiovascular del CMN 20 de Noviembre del ISSSTE. Rev Esp Med Quir. 2011;16:208-214. Disponible en: https://www.medigraphic.com/pdfs/quirurgicas/rmq2011/rmq114d.pdf

33. Reyes MH, Flores HS, Sauceda VAL, Vértiz RJdJ, Juárez RC, Wirtz VJ, et al. Percepción de los usuarios sobre la calidad de la atención ambulatoria en servicios de salud en México. Salud Publica Mex. 2013;55:S100-S105. Disponible en: http://www.scielo.org.mx/pdf/spm/ v55s2/v55s2a5.pdf

34. Martínez SS, Gómez HF, Lara GME. Percepción y cumplimiento del trato digno como indicador de calidad en la atención de enfermería en derechohabientes de una institución de salud. Horizonte Sanitario. 2015;14:96-100. Disponible en: http://www.redalyc.org/articulo. oa?id=457844966004

35. Doubova SV, Guanais FC, Pérez CR, Canning D, Macinko J, Reich MR. Attributes of patient-centered primary care associated with the public perception of good healthcare quality in Brazil, Colombia, Mexico and El Salvador. Health Policy Plan. 2016;31:834-843. Available from: https://doi.org/10.1093/heapol/czv139

36. Matas A. Diseño del formato de escalas tipo Likert: un estado de la cuestión. Revista Electrónica de Investigación Educativa. 2018;20:3847. Disponible en: https://doi.org/10.24320/redie.2018.20.1.1347

37. Castellanos-Olivares A, Fernández-García JO, Vásquez-Márquez PI. Mejorando la calidad de la atención anestésica en el paciente geriátrico. Rev Mex Anest. 2018;41:48-52. Disponible en: http://www.medigraphic. com/pdfs/rma/cma-2018/cmas181s.pdf

38. Covarrubias-Gómez A. Indicadores de calidad en la práctica de la anestesiología. Rev Mex Anest. 2018;41:79-82. Disponible en: http:// www.medigraphic.com/pdfs/rma/cma-2018/cma182a.pdf

39. Ugalde MC, Soto RB, Rosas BJV, Trujillo EC. Validación de un instrumento para evaluar la satisfacción del usuario durante el período preanestésico en el Hospital Regional $1^{\circ}$ de Octubre. Rev Esp Med Quir. 2012;17:44-50. Disponible en: https://www.medigraphic.com/ pdfs/quirurgicas/rmq-2012/rmq121j.pdf

40. Torres-González CA, Valera-Rodríguez Y, Pinto Segura-María E. Calidad de la atención en el servicio de anestesiología. Percepción del usuario. Acta Med. 2017;15:92-98. Disponible en: http://www.medigraphic.com/ pdfs/actmed/am-2017/am172b.pdf 